University of Texas at El Paso

ScholarWorks@UTEP

4-2007

\title{
Towards a General Description of Interval Multiplications: Algebraic Analysis and its Relation to T-Norms
}

Olga Kosheleva

The University of Texas at El Paso, olgak@utep.edu

Guenter Mayer

Vladik Kreinovich

The University of Texas at El Paso, vladik@utep.edu

Follow this and additional works at: https://scholarworks.utep.edu/cs_techrep

Part of the Computer Engineering Commons

Comments:

Technical Report: UTEP-CS-07-20

Published in: Marek Reformat and Michael R. Berthold (eds.), Proceedings of the 26th International Conference of the North American Fuzzy Information Processing Society NAFIPS'2007, San Diego, California, June 24-27, 2007, pp. 543-548.

\section{Recommended Citation}

Kosheleva, Olga; Mayer, Guenter; and Kreinovich, Vladik, "Towards a General Description of Interval Multiplications: Algebraic Analysis and its Relation to T-Norms" (2007). Departmental Technical Reports (CS). 141.

https://scholarworks.utep.edu/cs_techrep/141

This Article is brought to you for free and open access by the Computer Science at ScholarWorks@UTEP. It has been accepted for inclusion in Departmental Technical Reports (CS) by an authorized administrator of ScholarWorks@UTEP.For more information, please contact Iweber@utep.edu. 


\section{Towards a General Description of Interval Multiplications: Algebraic Analysis and Its Relation to t-Norms}

\author{
Olga Kosheleva \\ Department of Teacher Education \\ University of Texas at El Paso \\ El Paso, TX 79968, USA \\ olgak@utep.edu
}

\author{
Günter Mayer \\ Fachbereich Mathematik \\ Universität Rostock \\ D-18051 Rostock, Germany \\ guenter.mayer@uni-rostock.de
}

\author{
Vladik Kreinovich \\ Department of Computer Science \\ University of Texas at E1 Paso \\ El Paso, TX 79968, USA \\ vladik@utep.edu
}

\begin{abstract}
It is well known that interval computations are very important, both by themselves (as a method for processing data known with interval uncertainty) and as a way to process fuzzy data. In general, the problem of computing the range of a given function under interval uncertainty is computationally difficult (NP-hard). As a result, there exist different methods for estimating such a range: some methods require a longer computation time and lead to more accurate results, other methods lead to somewhat less accurate results but are much faster than the more accurate techniques. In particular, different methods exist for interval multiplication, i.e., for computing the range of a product of two numbers known with interval uncertainty. To select a method which is the best in a given situation, it is desired to be able to describe all possible methods. In this paper, we provide a description of all possible operations for interval multiplication; this description is based on the same ideas as a known description of t-norms in fuzzy logic.
\end{abstract}

\section{NeEd for Data Processing}

In many real-life situations, we are interested in the value of a physical quantity $y$ that is difficult or impossible to measure directly. Examples of such quantities are the distance to a star and the amount of oil in a given well. Since we cannot measure $y$ directly, a natural idea is to measure $y$ indirectly. Specifically, we find some easier-to-measure quantities $x_{1}, \ldots, x_{n}$ which are related to $y$ by a known relation $y=f\left(x_{1}, \ldots, x_{n}\right)$; this relation may be a simple functional transformation, or complex algorithm (e.g., for the amount of oil, numerical solution to an inverse problem). Then, to estimate $y$, we first measure the values of the quantities $x_{1}, \ldots, x_{n}$, and then we use the results $\widetilde{x}_{1}, \ldots, \widetilde{x}_{n}$ of these measurements to to compute an estimate $\widetilde{y}$ for $y$ as $\widetilde{y}=f\left(\widetilde{x}_{1}, \ldots, \widetilde{x}_{n}\right)$ :

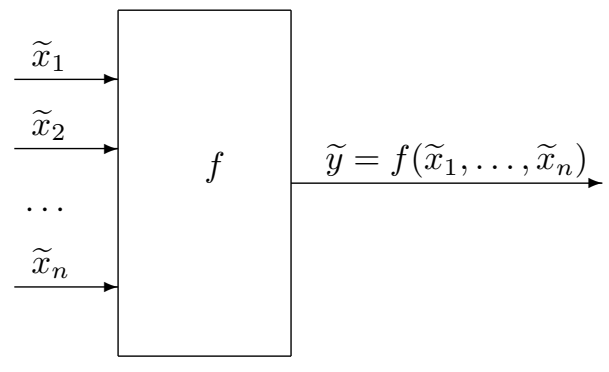

For example, to find the resistance $R$, we measure current $I$ and voltage $V$, and then use the known relation $R=V / I$ to estimate resistance as $\widetilde{R}=\widetilde{V} / \widetilde{I}$.

Computing an estimate for $y$ based on the results of direct measurements is called data processing; data processing is the main reason why computers were invented in the first place, and data processing is still one of the main uses of computers as number crunching devices.

Comment. In this paper, for simplicity, we consider the case when the relation between $x_{i}$ and $y$ is known exactly; in some practical situations, we only known an approximate relation between $x_{i}$ and $y$.

\section{Why Interval Computations? From Computing TO PROBABILITIES TO INTERVALS}

Measurements are never $100 \%$ accurate, so in reality, the actual value $x_{i}$ of $i$-th measured quantity can differ from the measurement result $\widetilde{x}_{i}$. Because of these measurement errors $\Delta x_{i} \stackrel{\text { def }}{=} \widetilde{x}_{i}-x_{i}$, the result $\widetilde{y}=f\left(\widetilde{x}_{1}, \ldots, \widetilde{x}_{n}\right)$ of data processing is, in general, different from the actual value $y=f\left(x_{1}, \ldots, x_{n}\right)$ of the desired quantity $y$.

It is desirable to describe the error $\Delta y \stackrel{\text { def }}{=} \widetilde{y}-y$ of the result of data processing. To do that, we must have some information about the errors of direct measurements.

What do we know about the errors $\Delta x_{i}$ of direct measurements? First, the manufacturer of the measuring instrument must supply us with an upper bound $\Delta_{i}$ on the measurement error. If no such upper bound is supplied, this means that no accuracy is guaranteed, and the corresponding "measuring instrument" is practically useless. In this case, once we performed a measurement and got a measurement result $\widetilde{x}_{i}$, we know that the actual (unknown) value $x_{i}$ of the measured quantity belongs to the interval $\mathbf{x}_{i}=\left[\underline{x}_{i}, \bar{x}_{i}\right]$, where $\underline{x}_{i}=\widetilde{x}_{i}-\Delta_{i}$ and $\bar{x}_{i}=\widetilde{x}_{i}+\Delta_{i}$.

In many practical situations, we not only know the interval $\left[-\Delta_{i}, \Delta_{i}\right]$ of possible values of the measurement error; we also know the probability of different values $\Delta x_{i}$ within this interval. This knowledge underlies the traditional engineering 
approach to estimating the error of indirect measurement, in which we assume that we know the probability distributions for measurement errors $\Delta x_{i}$.

In practice, we can determine the desired probabilities of different values of $\Delta x_{i}$ by comparing the results of measuring with this instrument with the results of measuring the same quantity by a standard (much more accurate) measuring instrument. Since the standard measuring instrument is much more accurate than the one use, the difference between these two measurement results is practically equal to the measurement error; thus, the empirical distribution of this difference is close to the desired probability distribution for measurement error. There are two cases, however, when this determination is not done:

- First is the case of cutting-edge measurements, e.g., measurements in fundamental science. When a Hubble telescope detects the light from a distant galaxy, there is no "standard" (much more accurate) telescope floating nearby that we can use to calibrate the Hubble: the Hubble telescope is the best we have.

- The second case is the case of measurements on the shop floor. In this case, in principle, every sensor can be thoroughly calibrated, but sensor calibration is so costly - usually costing ten times more than the sensor itself that manufacturers rarely do it.

In both cases, we have no information about the probabilities of $\Delta x_{i}$; the only information we have is the upper bound on the measurement error.

In this case, after we performed a measurement and got a measurement result $\widetilde{x}_{i}$, the only information that we have about the actual value $x_{i}$ of the measured quantity is that it belongs to the interval $\mathbf{x}_{i}=\left[\widetilde{x}_{i}-\Delta_{i}, \widetilde{x}_{i}+\Delta_{i}\right]$. In such situations, the only information that we have about the (unknown) actual value of $y=f\left(x_{1}, \ldots, x_{n}\right)$ is that $y$ belongs to the range $\mathbf{y}=[\underline{y}, \bar{y}]$ of the function $f$ over the box $\mathbf{x}_{1} \times \ldots \times \mathbf{x}_{n}$ :

$$
\mathbf{y}=[\underline{y}, \bar{y}]=\left\{f\left(x_{1}, \ldots, x_{n}\right) \mid x_{1} \in \mathbf{x}_{1}, \ldots, x_{n} \in \mathbf{x}_{n}\right\} .
$$

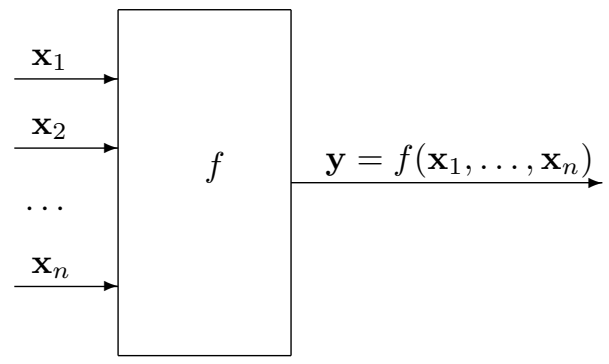

The process of computing this interval range based on the input intervals $\mathbf{x}_{i}$ is called interval computations; see, e.g., [6], [12].

\section{CASE OF FUZZY UNCERTAINTY: REMINDER}

Often, knowledge comes in terms of uncertain expert estimates. In the fuzzy case, to describe this uncertainty, for each value of estimation error $\Delta x_{i}$, we describe the degree $\mu_{i}\left(\Delta x_{i}\right)$ to which this value is possible.
For each degree of certainty $\alpha$, we can determine the set of values of $\Delta x_{i}$ that are possible with at least this degree of certainty - the $\alpha$-cut $\{x \mid \mu(x) \geq \alpha\}$ of the original fuzzy set. In most cases, this $\alpha$-cut is an interval.

Vice versa, if we know $\alpha$-cuts for every $\alpha$, then, for each object $x$, we can determine the degree of possibility that $x$ belongs to the original fuzzy set [2], [8], [13], [15], [16]. A fuzzy set can be thus viewed as a nested family of its $\alpha$-cuts.

So, if instead of a (crisp) interval $\mathbf{x}_{i}$ of possible values of the measured quantity, we have a fuzzy set $\mu_{i}(x)$ of possible values, then we can view this information as a family of nested intervals $\mathbf{x}_{i}(\alpha)-\alpha$-cuts of the given fuzzy sets.

\section{From the Computational Viewpoint, PROCESSING FuZZY UnCERTAINTY REDUCES TO PROCESSING OF INTERVAL UNCERTAINTY}

We have already mentioned that if instead of a (crisp) interval $\mathbf{x}_{i}$ of possible values of the measured quantity, we have a fuzzy set $\mu_{i}(x)$ of possible values, then we can view this information as a family of nested intervals $\mathbf{x}_{i}(\alpha)-\alpha$-cuts of the given fuzzy sets.

Our objective is then to compute the fuzzy number corresponding to this the desired value $y=f\left(x_{1}, \ldots, x_{n}\right)$. In this case, for each level $\alpha$, to compute the $\alpha$-cut of this fuzzy number, we can apply interval computations to the $\alpha$-cuts $\mathbf{x}_{i}(\alpha)$ of the corresponding fuzzy sets. The resulting nested intervals form the desired fuzzy set for $y$.

So, e.g., if we want to describe 10 different levels of uncertainty, then we must solve 10 interval computation problems.

Comment. In many practical situations, there is no need to perform 10 computations. For example, it is often reasonable to assume that the processing function is linear (i.e., terms quadratic in $\Delta x_{i}$ can be safely ignored) and all the membership functions $\mu_{i}\left(x_{i}\right)$ have the same shape and only differ by a scaling parameter, i.e., all have the form $\mu_{i}\left(\Delta x_{i}\right)=$ $\mu_{0}\left(\Delta x_{i} / \Delta_{i}\right)$ for some fixed function $\mu_{0}(x)$ (e.g., triangular or Gaussian). In this case, as it is well known [3], [4], [5], [7], the membership function for $\Delta y$ has a similar form $\mu_{0}(\Delta y / \Delta)$, where $\Delta$ is determined by the applying interval computations to the largest intervals (corresponding to $\alpha=0$ ).

\section{InTERVAl COMPUTATIONS TECHNIQUES: BRIEF REMINDER}

Historically the first method for computing the enclosure for the range is the method which is sometimes called "straightforward" interval computations. This method is based on the fact that inside the computer, every algorithm consists of elementary operations (arithmetic operations, min, max, etc.). For each elementary operation $f(a, b)$, if we know the intervals $\mathbf{a}$ and $\mathbf{b}$ for $a$ and $b$, we can compute the exact range $f(\mathbf{a}, \mathbf{b})$. The corresponding formulas form the so-called interval arithmetic. For example,

$[\underline{a}, \bar{a}]+[\underline{b}, \bar{b}]=[\underline{a}+\underline{b}, \bar{a}+\bar{b}] ; \quad[\underline{a}, \bar{a}]-[\underline{b}, \bar{b}]=[\underline{a}-\bar{b}, \bar{a}-\underline{b}] ;$
$[\underline{a}, \bar{a}] \cdot[\underline{b}, \bar{b}]=[\min (\underline{a} \cdot \underline{b}, \underline{a} \cdot \bar{b}, \bar{a} \cdot \underline{b}, \bar{a} \cdot \bar{b}), \max (\underline{a} \cdot \underline{b}, \underline{a} \cdot \bar{b}, \bar{a} \cdot \underline{b}, \bar{a} \cdot \bar{b})]$. 
In straightforward interval computations, we repeat the computations forming the program $f$ step-by-step, replacing each operation with real numbers by the corresponding operation of interval arithmetic. It is known that, as a result, we get an enclosure $\mathbf{Y} \supseteq \mathbf{y}$ for the desired range.

In some cases, this enclosure is exact. In more complex cases (see examples below), the enclosure has excess width.

\section{EXAMPLE}

Let us illustrate the above idea on the example of estimating the range of the function $f(x)=(x-2) \cdot(x+2)$ on the interval $x \in[1,2]$.

We start with parsing the expression for the function, i.e., describing how a computer will compute this expression; it will implement the following sequence of elementary operation:

$$
r_{1}:=x-2 ; \quad r_{2}:=x+2 ; \quad r_{3}:=r_{1} \cdot r_{2} .
$$

The main idea behind straightforward interval computations is to perform the same operations, but with intervals instead of numbers:

$$
\begin{gathered}
\mathbf{r}_{1}:=[1,2]-[2,2]=[-1,0] ; \quad \mathbf{r}_{2}:=[1,2]+[2,2]=[3,4] ; \\
\mathbf{r}_{3}:=[-1,0] \cdot[3,4]=[-4,0] .
\end{gathered}
$$

For this function, the actual range is $f(\mathbf{x})=[-3,0]$.

Comment: this is just a toy example, there are more efficient ways of computing an enclosure $\mathbf{Y} \supseteq \mathbf{y}$.

\section{More Sophisticated InTERVAl COMPUTATIONS TECHNIQUES: CENTERED FORM}

A better range is often provided by a centered form, in which a range $f\left(\mathbf{x}_{1}, \ldots, \mathbf{x}_{n}\right)$ of a smooth function on a box $\mathbf{x}_{1} \times \ldots \times \mathbf{x}_{n}$ is estimated as

$$
\begin{gathered}
f\left(\mathbf{x}_{1}, \ldots, \mathbf{x}_{n}\right) \subseteq \\
f\left(\widetilde{x}_{1}, \ldots, \widetilde{x}_{n}\right)+\sum_{i=1}^{n} \frac{\partial f}{\partial x_{i}}\left(\mathbf{x}_{1}, \ldots, \mathbf{x}_{n}\right) \cdot\left[-\Delta_{i}, \Delta_{i}\right],
\end{gathered}
$$

where $\widetilde{x}_{i} \stackrel{\text { def }}{=}\left(\underline{x}_{i}+\bar{x}_{i}\right) / 2$ is the interval's midpoint and $\Delta_{i} \stackrel{\text { def }}{=}$ $\left(\bar{x}_{i}-\underline{x}_{i}\right) / 2$ is its half-width.

The derivatives can be computed by using automatic differentiation techniques; see, e.g., [6].

Centered form provides an accuracy of order $O\left(\varepsilon^{2}\right)$, where $\varepsilon$ is the largest width of the input intervals.

\section{EXAMPLE}

For example, for the function $f(x)=(x-2) \cdot(x+2)$ on the interval $x \in[1,2]$, we have only one variable $x$. With respect to this variable, the derivative is equal to $(x+2)+(x-2)$. The range of this derivative for $x \in[1,2]$ is equal to

$$
([1,2]-2)+([1,2]+2)=[-1,0]+[3,4]=[2,4],
$$

the midpoint is $\widetilde{x}=1.5$ (at which $f(\widetilde{x})=-1.75$ ), and the halfwidth is $\Delta=0.5$. Thus, the resulting estimate for the range is

$-1.75+[2,4] \cdot[-0.5,0.5]=-1.75+[-2,2]=[-3.75,0.25]$.

\section{ANOTHER Interval IdEA: Bisection}

To get an even better estimate for the range, we can bisect the original interval(s), compute the ranges over all subintervals, and then take the union of the resulting ranges. For example, $f(x)=(x-2) \cdot(x+2)$ on the interval $x \in[1,2]$, after bisection, we get intervals $[1,1.5]$ and $[1.5,2]$. Over the first subinterval, the centered form leads to the range

$$
\begin{gathered}
\left(1.25^{2}-4\right)+[2,3] \cdot[-0.25,0.25]=1.5625-4+[-0.75,0.75]= \\
{[-3.1875,-1.6875] .}
\end{gathered}
$$

Over the second subinterval, the centered form leads to the range

$$
\begin{gathered}
\left(1.75^{2}-4\right)+[3,4] \cdot[-0.25,0.25]=3.0625-4+[-1,1]= \\
{[-1.9375,0.0625] .}
\end{gathered}
$$

Thus, the union of these two estimates is $[-3.1875,0.0625]$, which is much closer to the actual range $[-3,0]$.

If we apply bisection to each of these intervals, we get an even better estimate for the range.

\section{Interval Computations ARe Computationally EXPENSIVE}

In the above example, the more accurate range we want, the more computations we need to perform. This is not accidental: it turns out that in general, the problem of estimating the exact range

$$
[\underline{y}, \bar{y}]=f\left(\mathbf{x}_{1}, \ldots, \mathbf{x}_{n}\right)=\left\{f\left(x_{1}, \ldots, x_{n}\right): x_{i} \in\left[\underline{x}_{i}, \bar{x}_{i}\right]\right\}
$$

of an arithmetic expression $f\left(x_{1}, \ldots, x_{n}\right)$ under interval uncertainty $x_{i} \in\left[\underline{x}_{i}, \bar{x}_{i}\right]$ is computationally difficult (NP-hard); see, e.g., [10].

\section{Interval Computations: Need for Trade-OfF BetweEn ACCURACY AND COMPUTATION TIME}

Crudely speaking, NP-hardness means that for problems of reasonable size, exact computation is practically impossible. For such problems, we need techniques for approximate computations.

As a result, there exist different methods for estimating such a range: some methods require a longer computation time and lead to more accurate results, other methods lead to somewhat less accurate results but are much faster than the more accurate techniques.

\section{XiI. Particular Case of the Trade-Off: Different OPERATION FOR INTERVAL MULTIPLICATION}

In this paper, we will consider different operations for interval multiplication. We have already mentioned that for multiplication $f\left(x_{1}, x_{2}\right)=x_{1} \cdot x_{2}$, there exists an exact formula $[y, \bar{y}]$, where

$$
\underline{y}=\min \left(\underline{x}_{1}, \underline{x}_{2}, \underline{x}_{1}, \bar{x}_{2}, \bar{x}_{1}, \underline{x}_{2}, \bar{x}_{1}, \bar{x}_{2}\right)
$$

and

$$
\bar{y}=\max \left(\underline{x}_{1}, \underline{x}_{2}, \underline{x}_{1}, \cdot \bar{x}_{2}, \bar{x}_{1}, \underline{x}_{2}, \bar{x}_{1}, \bar{x}_{2}\right) .
$$


In addition to this exact formula, there are also faster-tocompute approximate formulas: e.g., to estimate the range of $y=x_{1} \cdot x_{2}$ when

$$
x_{1} \in\left[\widetilde{x}_{1}-\Delta_{1}, \widetilde{x}_{1}+\Delta_{1}\right] \text { and } x_{2} \in\left[\widetilde{x}_{2}-\Delta_{2}, \widetilde{x}_{2}+\Delta_{2}\right],
$$

we can find the smallest $\Delta$ for which all possible values of $x_{1} \cdot x_{2}$ belong to the interval $[\widetilde{y}-\Delta, \widetilde{y}+\Delta]$, where $\widetilde{y} \stackrel{\text { def }}{=} \widetilde{x}_{1} \cdot \widetilde{x}_{2}$.

The resulting interval arithmetic has been successfully used, e.g., in [17]. In particular, for intervals of non-negative numbers, we get $\Delta=\widetilde{x}_{1} \cdot \Delta_{2}+\widetilde{x}_{2} \cdot \Delta_{2}+\Delta_{1} \cdot \Delta_{2}$.

Similar formulas have been used for complex arithmetic [17].

Other interval multiplication operations have also been proposed and successfully used.

\section{NEED FOR DESCRIPTION OF DIFFERENT INTERVAL MULTIPLICATION OPER ATIONS}

Different interval multiplication operations have their own advantages and drawbacks on the trade-off scale between accuracy and computational efficiency. To make effective decisions on selecting an operation, it is desirable to get a good general description of all possible interval multiplication operations.

\section{WHAT IS AN INTERVAL MULTIPLICATION OPERATION? MAIN REQUIREMENTS}

The following are reasonable requirements on the interval multiplication:

- this operation is commutative, i.e.,

$$
\mathbf{a} \cdot \mathbf{b}=\mathbf{b} \cdot \mathbf{a}
$$

for all possible intervals $\mathbf{a}$ and $\mathbf{b}$;

- this operation is associative, i.e.,

$$
\mathbf{a} \cdot(\mathbf{b} \cdot \mathbf{c})=(\mathbf{a} \cdot \mathbf{b}) \cdot \mathbf{c}
$$

for all possible intervals $\mathbf{a}, \mathbf{b}$, and $\mathbf{c}$;

- this operation is $\subseteq$-monotonic in the sense that if $\mathbf{a} \subseteq \mathbf{a}^{\prime}$ and $\mathbf{b} \subseteq \mathbf{b}^{\prime}$ then $\mathbf{a} \cdot \mathbf{b} \subseteq \mathbf{a}^{\prime} \cdot \mathbf{b}^{\prime}$;

- this operation produces an exact range when one of the intervals is a number (i.e., a degenerate interval): e.g., for $a>0$, we have $[a, a] \cdot[\underline{b}, \bar{b}]=[a \cdot \underline{b}, a \cdot \bar{b}]$.

\section{A 1-D Analogue of This Problem and T-Norms}

A similar 1-D problem of classifying all associative operations $a * b$ on real numbers (under reasonable monotonicity assumptions) is well known in the foundations of fuzzy techniques: it has led to a known classification of t-norms; see e.g., [8], [16].

Reminder: a t-norm is defined as a commutation associative monotonic operation $t:[0,1] \times[0,1] \rightarrow[0,1]$ for which $t(1,1)=1$ and $t(0,0)=t(0,1)=0$. A t-norm is a fuzzy analog of an "and" operation: if our degree of trust in $A$ is $a$, and our degree of trust in $B$ is $b$, then our degree of trust in " $A$ and $B$ " is $t(a, b)$. Since " $A$ and $B$ " means the same as " $B$ and $A "$, it is reasonable to require that $t(a, b)=t(b, a)$; this explain commutativity. Other properties can be justified in a similar way.
According to the known classification, some t-norms can be represented as $a * b=F^{-1}(F(a)+F(b))$ for an appropriate function $F(x)$ (where $F^{-1}$ denotes an inverse function), and all the others (such as $a * b=\min (a, b)$ ) can be represented as limits of such t-norms. For example,

$$
\min (a, b)=\lim _{t \rightarrow \infty}\left(a^{-t}+b^{-t}\right)^{-1 / t},
$$

i.e., it is equal to the limit of expressions corresponding to $F(a)=a^{-t}$.

The above formula can be reformulated as $F(a * b)=$ $F(a)+F(b)$, i.e., as a fact that the operation $*$ is isomorphic to addition.

A similar classification is known for commutative associative monotonic operations defined on arbitrary subintervals of the real line; see, e.g., [14]

\section{The Complexity of OUR 2-D Classification PROBLEM}

The main difficulty in describing possible interval multiplication operations is that, in contrast to operations on the 1-D set of real numbers, there is no known classification result for associative operations on the 2-D set of intervals.

\section{How We Solve This Problem}

In general, we need two functions of four variables. By definition, an interval multiplication operation is a function that maps a pair of intervals $\left[\underline{x}_{1}, \bar{x}_{1}\right]$ and $\left[\underline{x}_{2}, \bar{x}_{2}\right]$ into a new interval $[y, \bar{y}]$. Every interval is characterized by two real-valued components (its endpoints). In terms of these components, both $y$ and $\bar{y}$ are functions of four input variables $\underline{x}_{1}, \bar{x}_{1}, \underline{x}_{2}$, and $\bar{x}_{2}$. Thus, to describe a general interval multiplication operation, we need to describe two functions of four variables.

Reduction to two functions of two variables. Every interval $\mathbf{x}_{i}=\left[\widetilde{x}_{i}-\Delta_{i}, \widetilde{x}_{i}+\Delta_{i}\right]$ with $\widetilde{x}_{i}>0$ can be represented as a product of its midpoint $\widetilde{x}_{i}$ and the interval

$$
I\left(\delta_{i}\right) \stackrel{\text { def }}{=}\left[1-\delta_{i}, 1+\delta_{i}\right]
$$

for $\delta_{i} \stackrel{\text { def }}{=} \Delta_{i} / \widetilde{x}_{i}$. This value $\delta_{i}$ is called a relative half-width of the interval $\mathbf{x}_{i}$. Due to commutativity and associativity, we thus have

$$
\mathbf{x}_{1} \cdot \mathbf{x}_{2}=\left(\widetilde{x}_{1} \cdot \widetilde{x}_{2}\right) \cdot\left[I\left(\delta_{1}\right) \cdot I\left(\delta_{2}\right)\right] .
$$

By the properties of the interval multiplication, the product of an interval $I\left(\delta_{1}\right) \cdot I\left(\delta_{2}\right)$ and a real number $\widetilde{x}_{1} \cdot \widetilde{x}_{2}$ is equal to the actual range. Thus, to describe a general interval multiplication operation, it is sufficient to describe the product $I\left(\delta_{1}\right) \cdot I\left(\delta_{2}\right)$ of two intervals $I\left(\delta_{1}\right) \cdot I\left(\delta_{2}\right)$ of type $\left[1-\delta_{i}, 1+\delta_{i}\right]$.

To describe this product, we must describe the two endpoints of this product interval as functions of two variables $\delta_{1}$ and $\delta_{2}$. So, to describe a general interval multiplication operation, we need two real-valued functions of two real variables. 
Examples. For the standard interval multiplication, when $\delta_{i}<$ 1 , we have

$$
\begin{gathered}
I\left(\delta_{1}\right) \cdot I\left(\delta_{2}\right)=\left[1-\delta_{1}, 1+\delta_{1}\right] \cdot\left[1-\delta_{2}, 1+\delta_{2}\right]= \\
{\left[\left(1-\delta_{1}\right) \cdot\left(1-\delta_{2}\right),\left(1+\delta_{1}\right) \cdot\left(1+\delta_{2}\right)\right] .}
\end{gathered}
$$

For Rump's arithmetic from [17],

$$
\begin{gathered}
I\left(\delta_{1}\right) \cdot I\left(\delta_{2}\right)=\left[1-\delta_{1}, 1+\delta_{1}\right] \cdot\left[1-\delta_{2}, 1+\delta_{2}\right]= \\
{\left[1-\left(\delta_{1}+\delta_{2}+\delta_{1} \cdot \delta_{2}\right), 1+\left(\delta_{1}+\delta_{2}+\delta_{1} \cdot \delta_{2}\right)\right],}
\end{gathered}
$$

i.e., $I\left(\delta_{1}\right) \cdot I\left(\delta_{2}\right)=I(\delta)$, where $\delta=\delta_{1}+\delta_{2}+\delta_{1} \cdot \delta_{2}$.

Reduction to one function of two variables and one function of one variable. We achieved the above reduction by representing each input interval $\mathbf{x}_{i}$ as a product of its midpoint $\widetilde{x}_{i}$ and an interval $I\left(\delta_{i}\right)$ centered at 1 . To get a further reduction, let us apply this representation to the output interval.

We have already mentioned that it is sufficient to consider the products $I\left(\delta_{1}\right) \cdot I\left(\delta_{2}\right)$. For this product, a similar representation takes the form

$$
I\left(\delta_{1}\right) \cdot I\left(\delta_{2}\right)=m\left(\delta_{1}, \delta_{2}\right) \cdot I\left(w\left(\delta_{1}, \delta_{2}\right)\right),
$$

where $m\left(\delta_{1}, \delta_{2}\right)$ denotes the midpoint of the product interval $I\left(\delta_{1}\right) \cdot I\left(\delta_{2}\right)$, and $w\left(\delta_{1}, \delta_{2}\right)$ denotes the relative half-width of this product interval.

Since the relative half-width does not change if we multiply an interval by a number, this means, in particular, that the relative half-width $\delta$ of the product $\mathbf{y}=\mathbf{x}_{1} \cdot \mathbf{x}_{2}$ of the two intervals $\mathbf{x}_{i}$ depends only on the relative half-widths $\delta_{i}$ of the corresponding intervals: $\delta=w\left(\delta_{1}, \delta_{2}\right)$.

As a result, when we compute the product

$$
\left(I\left(\delta_{1}\right) \cdot I\left(\delta_{2}\right)\right) \cdot I\left(\delta_{3}\right),
$$

the resulting relative half-width is equal to $w\left(w\left(\delta_{1}, \delta_{2}\right), \delta_{3}\right)$. So, the associativity of the interval multiplication means that $w$ is an associate operation on real numbers. Similarly, commutativity of the interval multiplication means that $w$ is commutative, and monotonicity means that $w$ is monotonic.

Thus, we can use the known classification result and conclude that either $w(a, b)=F^{-1}(F(a)+F(b))$ for an appropriate function $F(x)$, or $w$ is a limit of such functions. Thus, in the generic case (if we ignore the limit cases)

$$
I\left(\delta_{1}\right) \cdot I\left(\delta_{2}\right)=m\left(\delta_{1}, \delta_{2}\right) \cdot I\left(F^{-1}\left(F\left(\delta_{1}\right)+F\left(\delta_{2}\right)\right)\right) .
$$

In other words, to describe a general interval multiplication operation, we need a function $m\left(\delta_{1}, \delta_{2}\right)$ of two variables and a function $F(a)$ of one variable.

Examples. For the standard interval multiplication,

$$
\begin{gathered}
m\left(\delta_{1}, \delta_{2}\right)=\frac{\left(1-\delta_{1}\right) \cdot\left(1-\delta_{2}\right)+\left(1+\delta_{1}\right) \cdot\left(1+\delta_{2}\right)}{2}= \\
1+\delta_{1} \cdot \delta_{2},
\end{gathered}
$$

and therefore

$$
w\left(\delta_{1}, \delta_{2}\right)=\frac{\delta_{1}+\delta_{2}}{1+\delta_{1} \cdot \delta_{2}} .
$$

(Incidentally, this is the formula for adding velocities in Special Relativity theory, if we express velocities in units in which the speed of light $c$ is 1.) It is known that for the hyperbolic tangent $\tanh (x)$, we have

$$
\tanh (a+b)=\frac{\tanh (a)+\tanh (b)}{1+\tanh (a) \cdot \tanh (b)} ;
$$

thus, $w\left(\delta_{1}, \delta_{2}\right)=F^{-1}\left(F\left(\delta_{1}\right)+F\left(\delta_{2}\right)\right)$ where $F(a)$ is an inverse function to tanh, i.e.,

$$
F(a)=\tanh ^{-1}(a)=\frac{1}{2} \cdot \ln \left(\frac{1+a}{1-a}\right) .
$$

For Rump's arithmetic, $m\left(\delta_{1}, \delta_{2}\right)=1$, and $\delta=w\left(\delta_{1}, \delta_{2}\right)=$ $\delta_{1}+\delta_{2}+\delta_{1} \cdot \delta_{2}$. Here, $1+\delta=\left(1+\delta_{1}\right) \cdot\left(1+\delta_{2}\right)$, hence $\ln (1+\delta)=\ln \left(1+\delta_{1}\right)+\ln \left(1+\delta_{2}\right)$, and so $w\left(\delta_{1}, \delta_{2}\right)=$ $F^{-1}\left(F\left(\delta_{1}\right)+F\left(\delta_{2}\right)\right)$ for $F(a)=\ln (1+a)$.

Reduction to two functions of one variable. For the above operation, $I\left(\delta_{1}\right) \cdot I\left(\delta_{2}\right)=m\left(\delta_{1}, \delta_{2}\right) \cdot I\left(\delta_{1} * \delta_{2}\right)$, where we denoted $\left.\delta_{1} * \delta_{2} \stackrel{\text { def }}{=} F^{-1}\left(F\left(\delta_{1}\right)+F\left(\delta_{2}\right)\right)\right)$. Thus,

$$
\begin{gathered}
\left(I\left(\delta_{1}\right) \cdot I\left(\delta_{2}\right)\right) \cdot I\left(\delta_{3}\right)=m\left(\delta_{1}, \delta_{2}\right) \cdot\left(I\left(\delta_{1} * \delta_{2}\right) \cdot I\left(\delta_{3}\right)\right)= \\
m\left(\delta_{1}, \delta_{2}\right) \cdot m\left(\delta_{1} * \delta_{2}, \delta_{3}\right) \cdot I\left(\delta_{1} * \delta_{2} * \delta_{3}\right) .
\end{gathered}
$$

So, associativity means that

$$
m\left(\delta_{1}, \delta_{2}\right) \cdot m\left(\delta_{1} * \delta_{2}, \delta_{3}\right)=m\left(\delta_{1}, \delta_{2} * \delta_{3}\right) \cdot m\left(\delta_{2}, \delta_{3}\right) .
$$

We know that the operation $*$ is simplified if we replace the original variables $\delta_{i}$ with the re-scaled ones $a_{i} \stackrel{\text { def }}{=} F\left(\delta_{i}\right)$ (so that $\left.\delta_{i}=F^{-1}\left(a_{i}\right)\right)$. In the new terms, the operation $\delta=\delta_{1} * \delta_{2}$ simply takes the form $a=a_{1}+a_{2}$.

In the new terms, instead of the original midpoint function $m\left(\delta_{1}, \delta_{2}\right)$, we consider a new function $M\left(a_{1}, a_{2}\right) \stackrel{\text { def }}{=} m\left(F^{-1}\left(\delta_{1}\right), F^{-1}\left(\delta_{1}\right)\right)$. For this new function, the associativity-related formula takes a simplified form

$$
M\left(a_{1}, a_{2}\right) \cdot M\left(a_{1}+a_{2}, a_{3}\right)=M\left(a_{1}, a_{2}+a_{3}\right) \cdot M\left(a_{2}, a_{3}\right) .
$$

If we take the logarithms of both side, then for $\ell\left(a_{1}, a_{2}\right) \stackrel{\text { def }}{=}$ $-\ln \left(M\left(a_{1}, a_{2}\right)\right)$, we get an even simpler expression

$$
\ell\left(a_{1}, a_{2}\right)+\ell\left(a_{1}+a_{2}, a_{3}\right)=\ell\left(a_{1}, a_{2}+a_{3}\right)+\ell\left(a_{2}, a_{3}\right) .
$$

In mathematical terms, this means that $\ell\left(a_{1}, a_{2}\right)$ is a 2 -cocycle in the sense of so called group cohomologies [11]. It is known that in this case, every co-cycle is a co-boundary [9], [11], i.e., $\ell\left(a_{1}, a_{2}\right)=g\left(a_{1}\right)+g\left(a_{2}\right)-g\left(a_{1}+a_{2}\right)$ for some function $g(a)$. So, for $M\left(a_{1}, a_{2}\right)=\exp \left(-\ell\left(a_{1}, a_{2}\right)\right)$, we have $M\left(a_{1}, a_{2}\right)=\frac{G\left(a_{1}+a_{2}\right)}{G\left(a_{1}\right) \cdot G\left(a_{2}\right)}$, for $G(a) \stackrel{\text { def }}{=} \exp (g(a))$. Substituting $a_{i}=F\left(a_{i}\right)$ into this expression, we conclude that $m\left(\delta_{1}, \delta_{2}\right)=\frac{\varphi\left(\delta_{1} * \delta_{2}\right)}{\varphi\left(\delta_{1}\right) \cdot \varphi\left(\delta_{2}\right)}$, where $\varphi(\delta) \stackrel{\text { def }}{=} G\left(F\left(\delta_{i}\right)\right)$.

Resulting general description of interval multiplication operations. Combining the above expressions for $m\left(\delta_{1}, \delta_{2}\right)$ and $w\left(\delta_{1}, \delta_{2}\right)$, we conclude that an arbitrary interval multiplication operation takes the form

$$
I\left(\delta_{1}\right) \cdot I\left(\delta_{2}\right)=\frac{\varphi\left(\delta_{1} * \delta_{2}\right)}{\varphi\left(\delta_{1}\right) \cdot \varphi\left(\delta_{2}\right)} \cdot I\left(\delta_{1} * \delta_{2}\right),
$$


where

$$
\delta_{1} * \delta_{2}=F^{-1}\left(F\left(\delta_{1}\right)+F\left(\delta_{2}\right)\right) .
$$

Thus, we need two monotonic functions of one variables $F(a)$ and $\varphi(\delta)$ to describe such an operation. (The actual interval multiplication operation can also be a limit of such operations.)

Comment. It is worth mentioning that a further reduction is impossible: one can check that for arbitrary monotonic functions $F(a)$ and $\varphi(\delta)$, the above formula defines an interval multiplication operation. For example, commutativity is straightforward, associativity comes from the fact that

$$
\begin{gathered}
\left(I\left(\delta_{1}\right) \cdot I\left(\delta_{2}\right)\right) \cdot I\left(\delta_{3}\right)=\frac{\varphi\left(\delta_{1} * \delta_{2}\right)}{\varphi\left(\delta_{1}\right) \cdot \varphi\left(\delta_{2}\right)} \cdot\left(I\left(\delta_{1} * \delta_{2}\right) \cdot I\left(\delta_{3}\right)\right)= \\
\frac{\varphi\left(\delta_{1} * \delta_{2}\right)}{\varphi\left(\delta_{1}\right) \cdot \varphi\left(\delta_{2}\right)} \cdot \frac{\varphi\left(\delta_{1} * \delta_{2} * \delta_{3}\right)}{\varphi\left(\delta_{1} * \delta_{2}\right) \cdot \varphi\left(\delta_{3}\right)} \cdot I\left(\delta_{1} * \delta_{2} * \delta_{3}\right)= \\
\frac{\varphi\left(\delta_{1} * \delta_{2} * \delta_{3}\right)}{\varphi\left(\delta_{1}\right) \cdot \varphi\left(\delta_{2}\right) \cdot \varphi\left(\delta_{3}\right)} \cdot I\left(\delta_{1} * \delta_{2} * \delta_{3}\right) .
\end{gathered}
$$

It is also worth mentioning that while usually, interval computations help in fuzzy computing, here, vice versa, a known fact from fuzzy theory helps interval computations.

\section{OTHER APplications of the Proposed ClassificATION}

Interval multiplication does not have to be an enclosure. We started with interval multiplication operations which provided an enclosure $\mathbf{Y} \supseteq \mathbf{y}$ for the actual interval $\mathbf{y}$, i.e., which guarantee that the product $y$ is within the resulting interval $\mathbf{Y}$. However, in our classification, we did not require that the result of the operation is an enclosure. Thus, this classification can also be applied to probabilistic situations, when we have intervals which contain $y$ only with a certain probability $\approx 1$.

Case of stochastic arithmetic. Such non-enclosure situations are described, e.g., by stochastic arithmetic (see, e.g., [1]), when for each variable $x_{i}$, we know the mean $\widetilde{x}_{i}$ and standard deviation $\sigma_{i}$. In statistics, we then usually conclude that the actual value $x_{i}$ is (with a high probability) located within an interval $\left[\widetilde{x}_{i}-k \cdot \sigma_{i}, \widetilde{x}_{i}+k \cdot \sigma_{i}\right]$, where $k=2,3$, or 6 depending on the desired level of certainty.

Interval multiplication for stochastic arithmetic. Within the stochastic arithmetic, we want, given the means $\widetilde{x}_{i}$ and standard deviations $\sigma_{i}$ of two variables $x_{1}$ and $x_{2}$, to find the mean $\widetilde{y}$ and the standard deviation $\sigma$ of their product. In stochastic arithmetic, the variables are assumed to be independent. For independent random variables, the expected value of a product is equal to the product of expected values, so $\widetilde{y}=E\left[x_{1} \cdot x_{2}\right]=E\left[x_{1}\right] \cdot E\left[x_{2}\right]=\widetilde{x}_{1} \cdot \widetilde{x}_{2}$. Similarly, $E\left[\left(x_{1} \cdot x_{2}\right)^{2}\right]=E\left[x_{1}^{2}\right] \cdot E\left[x_{2}^{2}\right]$; since $E\left[x_{i}^{2}\right]=\widetilde{x}_{i}^{2}+\sigma_{i}^{2}$, we conclude that $\widetilde{y}^{2}+\sigma^{2}=\left(\widetilde{x}_{1}^{2}+\sigma_{1}^{2}\right) \cdot\left(\widetilde{x}_{2}^{2}+\sigma_{2}^{2}\right)$. From this expression one can easily conclude that the corresponding multiplication operation is commutative, associative, monotonic, and consistent with multiplication by numbers.
Explicitly, the corresponding interval multiplication operation has the form $\widetilde{y}=\widetilde{x}_{1} \cdot \widetilde{x}_{2}$ and

$$
\sigma=\sqrt{\widetilde{x}_{1}^{2} \cdot \sigma_{2}^{2}+\widetilde{x}_{2}^{2} \cdot \sigma_{1}^{2}+\sigma_{1}^{2} \cdot \sigma_{2}^{2}} .
$$

This operation is a particular case of our general description. In this case, $m\left(\delta_{1}, \delta_{2}\right)=1$, and $w\left(\delta_{1}, \delta_{2}\right)=$ $\sqrt{1+\delta_{1}^{2}+\delta_{2}^{2}+\delta_{1}^{2} \cdot \delta_{2}^{2}}$. We have already mentioned that this expression is equivalent to $\left(1+\delta^{2}\right)=\left(1+\delta_{2}^{2}\right) \cdot\left(1+\delta_{2}^{2}\right)$, thus $\ln \left(1+\delta^{2}\right)=\ln \left(1+\delta_{2}^{2}\right)+\ln \left(1+\delta_{2}^{2}\right)$ and $w\left(\delta_{1}, \delta_{2}\right)=$ $F^{-1}\left(F\left(\delta_{1}\right)+F\left(\delta_{2}\right)\right)$ for $F(\delta)=\ln \left(1+\delta^{2}\right)$.

\section{ACKNOWLEDGMENTS}

This work was supported in part by NSF grants EAR0225670 and DMS-0532645 and by Texas Department of Transportation grant No. 0-5453.

The authors are thankful to the anonymous referees for important suggestions.

\section{REFERENCES}

[1] R. Alt, J.-L. Lamotte, and S. Markov, "Abstract structures in stochastic arithmetic", Proc. of the 11-th Conf. on Information Processing and Management of Uncertainties in Knowledge-based Systems IPMU'2006, Paris, France, July 2-7, 2006.

[2] G. Bojadziev and M. Bojadziev, Fuzzy sets, fuzzy logic, applications, World Scientific, Singapore, 1995.

[3] D. Dubois and H. Prade, "Operations on fuzzy numbers", International Journal of Systems Science, 1978, Vol. 9, pp. 613-626.

[4] D. Dubois and H. Prade, "Fuzzy real algebra: some results", Fuzzy Sets and Systems, 1979, Vol. 2, pp. 327-348.

[5] D. Dubois and H. Prade, Fuzzy sets and systems: theory and applications, Academic Press, New York, London, 1980.

[6] L. Jaulin, M. Kieffer, O. Didrit, and E. Walter, Applied interval analysis: with examples in parameter and state estimation, robust control and robotics, Springer Verlag, London, 2001.

[7] A. Kauffman and M. M. Gupta, Introduction to fuzzy arithmetic: theory and applications, Van Nostrand, New York, 1985.

[8] G. Klir and B. Yuan, Fuzzy sets and fuzzy logic, Prentice Hall, New Jersey, 1995.

[9] O. M. Kosheleva, "Axiomatization of volume in elementary geometry", Siberian Mathematical Journal, 1980, Vol. 21, pp. 78-85.

[10] V. Kreinovich, A. Lakeyev, J. Rohn, and P. Kahl, Computational complexity and feasibility of data processing and interval computations, Kluwer, Dordrecht, 1998.

[11] C. C. Moore, "Group extensions and cohomology for locally compact groups. III", Trans. Amer. Math, Soc., 1976, Vol. 221, No. 1, pp. 1-33.

[12] R. E. Moore, Automatic error analysis in digital computation, Technical Report Space Div. Report LMSD84821, Lockheed Missiles and Space Co., 1959.

[13] R. E. Moore and W. A. Lodwick, "Interval analysis and fuzzy set theory", Fuzzy Sets and Systems, 2003, Vol. 135, No. 1, pp. 5-9.

[14] P. S. Mostert and A. L. Shields, "On the structure of semigroups on a compact manifold with boundary," Ann. Math., 1957, Vol. 65, pp. 117143.

[15] H. T. Nguyen and V. Kreinovich, "Nested intervals and sets: concepts, relations to fuzzy sets, and applications", In: R. B. Kearfott and V. Kreinovich (eds), Applications of interval computations, Kluwer, Dordrecht, pp. 245-290.

[16] H. T. Nguyen and E. A. Walker, A First Course in Fuzzy Logic, CRC Press, Boca Raton, Florida, 2006.

[17] S. M. Rump, "Fast and parallel interval arithmetic", BIT, 1999, Vol. 39, No. 3, pp. 534-554. 